\title{
A mathematical model reveals the influence of population heterogeneity on herd immunity to SARS-CoV-2
}

\author{
Tom Britton ${ }^{1 *}$, Frank Ball ${ }^{2}$, Pieter Trapman' \\ ${ }^{1}$ Department of Mathematics, Stockholm University, Stockholm, Sweden. ${ }^{2}$ School of Mathematical Sciences, University of Nottingham, Nottingham, UK. \\ ${ }^{*}$ Corresponding author. E-mail:
}

Despite various levels of preventive measures, in $\mathbf{2 0 2 0}$ many countries have suffered severely from the coronavirus 2019 (COVID-19) pandemic caused by the severe acute respiratory syndrome coronavirus 2 (SARS-CoV-2) virus. We show that population heterogeneity can significantly impact disease-induced immunity as the proportion infected in groups with the highest contact rates is greater than in groups with low contact rates. We estimate that if $R_{0}=2.5$ in an age-structured community with mixing rates fitted to social activity then the disease-induced herd immunity level can be around $43 \%$, which is substantially less than the classical herd immunity level of $60 \%$ obtained through homogeneous immunization of the population. Our estimates should be interpreted as an illustration of how population heterogeneity affects herd immunity, rather than an exact value or even a best estimate.

Severe acute respiratory syndrome coronavirus 2 (SARS-CoV2) has spread globally despite the many different preventive measures that have been put in place to reduce transmission. Some countries aimed for suppression by extreme quarantine measures (lockdown), and others for mitigation by slowing the spread using certain preventive measures in combination with protection of the vulnerable (1). An important question for both policies has been when to lift some or all the restrictions. A closely related question is if and when herd immunity can be achieved. Herd immunity is defined as a level of population immunity such that disease spreading will decline and stop even after all preventive measures have been relaxed. If all preventive measures are relaxed when the immunity level from infection is below the herd immunity level, then a second wave of infection may start once restrictions are lifted.

By 1 May 2020, some regions and countries reached high estimates for the population immunity level, with for example $26 \%$ infected (and large confidence interval) in metropolitan Stockholm region, as based on a mathematical model (2). At the same time, population studies in Spain show that in second half of May over 10\% of the population of Madrid had antibodies for coronavirus disease 2019 (COVID-19) (3). It is debatable whether (classical) herd immunity for COVID-19, which is believed to lie between $50 \%$ and $75 \%$, can be achieved without unacceptably high case fatality rates (4-6).

The definition of classical herd immunity originates from mathematical models for the impact of vaccination. The classical herd immunity level $h_{\mathrm{C}}$ is defined as $h_{\mathrm{C}}=1-1 / R_{0}$, where $R_{0}$ is the basic reproduction number, defined as the average number of new infections caused by a typical infected individual during the early stage of an outbreak in a fully susceptible population (7). Thus, if a fraction $v$ is vaccinated (with a vaccine giving $100 \%$ immunity) and vaccinees are selected uniformly in the community, then the new reproduction number is $R_{v}=(1-v) R_{0}$. From this the critical vaccination coverage $v_{c}=1-1 / R$. So, if at least this fraction is vaccinated and hence immune, the community has reached herd immunity, as $R_{v} \leq 1$, and no outbreak can take place. If the vaccine is not perfect but instead reduces susceptibility by a fraction $E$ (so $E=1$ corresponds to $100 \%$ efficacy), then the critical vaccination coverage is given by $v_{c}=E^{-1}\left(1-1 / R_{0}\right)$ (7), implying that a bigger fraction needs to be vaccinated if the vaccine is not perfect.

No realistic model will depict human populations as homogenous, there are many heterogeneities in human societies that will influence virus transmission. Here, we illustrate how population heterogeneity can cause significant heterogeneity among the people infected during the course of an infectious disease outbreak, with consequent impact on the herd immunity level and the performance of exit policies aimed at minimizing the risk of future infection spikes.

One of the simplest of all epidemic models is to assume a homogeneously mixing population in which all individuals are equally susceptible, and equally infectious if they become infected. Before becoming infectious, infected individuals first go through a latent/exposed period, i.e., a SusceptibleExposed-Infected-Recovered (SEIR) model (7). The basic reproduction number $R_{0}$ denotes the average number of infectious contacts an infected individual has before recovering and becoming immune (or dying). An infectious contact is defined as one close enough to infect the other individual if this individual is susceptible (contacts with already infected individuals have no effect). 
To this simple model we add two important features known to play an important role in disease spreading (the model is described in full detail in the Supplementary material). The first is to include age structure by dividing the community into different age cohorts, with heterogeneous mixing between the different age cohorts. We categorize a community into six age groups and fit contact rates derived from an empirical study of social contacts (8) (see Supplementary material for details on the community structure). The person-toperson infectious contact rate between two individuals depends on the age groups of both individuals. The average number of infectious contacts an infected person in age group, $i$, has with individuals in (another or the same) age group, $j$, now equals $a_{i j} \pi_{j}$, where $a_{i j}$ reflects both how much an $i$-individual has contact with a specific $j$-individual. It also reflects the typical infectivity of $i$-individuals and susceptibility of $j$-individuals. The population fraction of individuals belonging to age cohort $j$ is denoted by $\pi_{j}$.

The second population structure element categorizes individuals according to their social activity level. A common way to do this is by means of network models (e.g., (9)). Here we take a simpler approach and categorize individuals into three different activity levels, which are arbitrary and chosen for illustration purposes: $50 \%$ of each age cohort have normal activity, $25 \%$ have low activity corresponding to half as many contacts compared to normal activity, and $25 \%$ have high activity corresponding to twice as many contacts as normal activity. By this we mean that, for example, a high-activity individual in age group $i$ on average has $2^{*} a_{i j} \pi_{j}^{*} 0.5^{*} 0.25$ infectious contacts with low-activity individuals of age group $j$. The factor 2 comes from the infective having high activity, the factor 0.5 from the contacted person having low activity, and the factor 0.25 from low-activity individuals making up $25 \%$ of each age cohort. The basic reproduction number $R_{0}$ for this model is given by the dominant eigenvalue of the (next-generation) matrix $M$ having these elements as its entries. (7).

The final fractions of the different groups in the population becoming infected in the epidemic are obtained by solving a set of equations (the final-size equations given in the supplementary material). To be able to say something about the time evolution of the epidemic we assume a classical SEIR epidemic model. More precisely, we assume that individuals who get infected are initially latent for a mean of 3 days, followed by an infectious period of a mean of 4 days, thus approximately mimicking the situation for COVID-19 (e.g., (1)). During the infectious period an individual makes infectious contacts at rates such that the mean numbers of infectious contacts agree with those of the next-generation matrix $M$.

We assume that the basic reproduction number satisfies $R_{0}=2.5$ (a few other values are also evaluated) and that the epidemic is initiated with a small fraction of infectious individuals on February 15. On March 15, when the fraction infected is still small, preventive measures are implemented such that all averages in the next-generation matrix are scaled by the same factor $\alpha<1$, so the next-generation matrix becomes $\alpha M$. Consequently, the new reproduction number is $\alpha R_{0}$. These preventive measures are kept until the ongoing epidemic is nearly finished. That is, all preventive measures are relaxed thus setting $\alpha$ back to 1 on June 30. If herd immunity is not reached there will then be a second wave, whereas if herd immunity has been achieved the epidemic continues to decline.

We used the model to investigate the effect of the preventive measures and for two scenarios we analyze whether or not a given level of preventive measures yields disease-induced herd immunity. We do this for populations that are (i) homogeneous, (ii) categorized by age groups but not by activity levels, (iii) not categorized by age but are assigned different activity levels, and (iv) have both age-related and activity structures.

For each of the four population structures described above, we show overall disease-induced herd immunity in Table 1. This was obtained by assuming that preventive measures having factor $\alpha<1$ are implemented at the start of an epidemic, running the resulting model epidemic to its conclusion and then exposing the population to a second epidemic with $\alpha=1$. We obtain $\alpha_{*}$, the greatest value of $\alpha$ such that a second epidemic cannot occur. The disease-induced herd immunity level $h_{\mathrm{D}}$ is given by the fraction of the population that is infected by the first epidemic. This approximates the situation where preventive measures are implemented early and lifted late in an outbreak. Note that $h_{\mathrm{D}}$, given the next-generation matrix, is independent of the distributions of the latent and infectious periods.

As seen in Table 1, all three structured populations have lower disease-induced herd immunity $h_{\mathrm{D}}$ compared to the classical herd immunity $h_{\mathrm{C}}$, which assumes immunity is uniformly distributed among the different types of individual. From the table it is clear that the different activity levels have a greater effect on reducing $h_{\mathrm{D}}$ than age structure.

In Table 2, the final fractions infected in the different age activity groups for $\alpha=\alpha_{*}$ just barely reaching disease-induced herd immunity are given. This is done for the age and activity group structure and assuming $R_{0}=2.5$. The overall fraction infected equals $h_{\mathrm{D}}=43.0 \%$, in agreement with Table 1 . Table $\mathrm{S} 1$ is a similar table where only activity groups are considered.

We also illustrate the time evolution of the epidemic for $R_{0}=2.5$, assuming both age and activity structure and starting with a small fraction externally infected in mid-February. For this we show the epidemic over time for four different levels of preventive measures put in place early in the epidemic outbreak (mid-March) and being relaxed once 
transmission has dropped to low levels (June 30). In Fig. 1, the community proportion that is infectious is plotted during the course of the epidemic.

On March 15 preventive measures (at four different levels for $\alpha$ ) are put in place and in every case the growth rate is reduced except when no preventative measures are applied (the blue curve; $\alpha=1$ ). Moreover, the preventive measures reduce the size of, and delay the time of, the peak. Sanctions are lifted on June 30 putting transmission rates back to their original levels, but only in the curve with highest sanctions is there a clear second wave, since the remaining curves have reached (close to) herd immunity. The yellow curve finishes below $50 \%$ getting infected. The reason this exceeds the $43 \%$ infected shown in Table 1 is that preventive measures were not imposed from the start and were lifted before the epidemic was over. The corresponding cumulative fraction infected as a function of time are shown in Fig. 2. An interesting observation is that the purple curve results in a higher overall fraction infected even though this scenario had more restrictions applied than the scenario of the yellow curve. This is because this epidemic was further from completion when sanctions were lifted.

Only the curve corresponding to greatest preventive measures shows a severe second wave when restrictions are lifted. In most cases no (strong) second wave of outbreak occurs once preventive measures are lifted. Note also that the yellow curve, in which the overall fraction infected is well below the classical herd immunity level $h_{\mathrm{C}}=60 \%$, is in fact protected by herd immunity since no second wave appears. See the supplement for depictions of when restrictions are lifted continuously between June 1 and August 31 (see figs. S1 and $\mathrm{S} 2$ ), and how the effective reproduction number evolves as a function of the time when restrictions are lifted (see fig. S3).

Our simple model shows how the disease-induced herd immunity level may be substantially lower than the classical herd immunity level derived from mathematical models assuming homogeneous immunization. Our application to COVID-19 indicates a reduction of herd immunity from $60 \%$ under homogeneous immunization down to $43 \%$ (assuming $R_{0}=2.5$ ) in a structured population, but this should be interpreted as an illustration, rather than an exact value or even a best estimate. To try to quantify more precisely the size of this effect remains to be done.

In our model we have taken age cohorts and social activity levels into account. However, more complex and realistic models have many other types of heterogeneities: for instance, increased spreading within households (of different sizes) or within schools and workplaces. Those activity levels and social structures are country or region specific and should be modeled as such. Further, spatial heterogeneity arises with rural areas having lower contact rates than metropolitan regions. It seems reasonable to assume that most such additional heterogeneities will have the effect of reducing the disease-induced immunity level $h_{\mathrm{D}}$ even further, in that high contact environments, such as metropolitan regions, large households and extensive, big workplaces for example will have a higher infected fraction and immunity will be concentrated even more among highly-active and connected individuals. Some complex models (e.g., (1)) categorize by, for example, age and spatial location but omit individual variation within each category. The latter can be incorporated by including different activity levels, or by adding a social network in which individuals have differing numbers of acquaintances. As we have illustrated, differences in social activity play a greater role in reducing the disease-induced herd immunity level than heterogeneous age-group mixing. Thus models excluding such features will see a smaller difference between $h_{\mathrm{D}}$ and $h_{\mathrm{C}}$. Our choice to have $50 \%$ having average activity, $25 \%$ having half and $25 \%$ having double activity is of course arbitrary. An important future task is to determine the size of differences in social activity within age groups for different types of populations. The greater the social heterogeneity there is between groups, the greater the difference between $h_{\mathrm{D}}$ and $h_{\mathrm{C}}$.

One assumption of our model is that preventive measures act proportionally on all contact rates. This may not always hold. For example, most countries aim to protect elderly and other risk groups, which does not obey this assumption. Again, we expect the effect of discriminatory protection would be to reduce the disease-induced immunity level, because the oldest age group has the fewest contacts. For a model including schools and workplaces, it is not obvious what effect school closure and strong recommendations to work from home would have on the disease-induced herd immunity level. A different model extension would be to allow individuals to change their activity level over time. The effect of such changes in activity levels, in particular if they vary between different categories, remains unknown.

In our model we assume that infection with and subsequent clearance of the virus leads to immunity against further infection for an extended period of time. If there is relatively quick loss of immunity or if we want to consider a time scale where the impact of demographic processes, such as births and people changing age-group becomes substantial, then we need further models.

Different forms of immunity levels have been discussed previously in the literature although, as far as we know, not when considering early-introduced preventions that are lifted toward the end of an epidemic outbreak. Anderson and May (10) concluded that immunity level may differ between uniformly distributed, disease-induced and optimally located immunity (see also (11)), and vaccination policies selecting individuals to immunize in an optimal manner have been discussed in many papers, e.g., (12). A recent independent paper 
by Gomes et al. (13) shows similar results to those of the present paper but considers heterogeneities in terms of continuously varying susceptibilities. That model is solved numerically similarly to our Fig. 1, but the analytical results for the final number of infected people and $h_{\mathrm{D}}$ are missing.

Rather than lifting all restrictions simultaneously, most countries are gradually lifting COVID-19 preventive measures. That strategy can avoid seeing the type of overshoot illustrated by the purple curve in Fig. 2, which results in a greater fraction infected than if milder restrictions are enacted (yellow curve).

\section{REFERENCES AND NOTES}

1. S. Flaxman, S. Mishra, A. Gandy, H. J. T. Unwin, H. Coupland, T. A. Mellan, H. Zhu, T. Berah, J. W. Eaton, P. N. P. Guzman, N. Schmit, L. Cilloni, K. E. C. Ainslie, M. Baguelin, I. Blake, A. Boonyasiri, O. Boyd, L. Cattarino, C. Ciavarella, L. Cooper, Z. Cucunubá, G. Cuomo-Dannenburg, A. Dighe, B. Djaafara, I. Dorigatti, S. van Elsland, R. FitzJohn, H. Fu, K. Gaythorpe, L. Geidelberg, N. Grassly, W. Green, T. Hallett, A. Hamlet, W. Hinsley, B. Jeffrey, D. Jorgensen, E. Knock, D. Laydon, G. Nedjati-Gilani, P. Nouvellet, K. Parag, I. Siveroni, H. Thompson, R. Verity, E. Volz, C. Walters, H. Wang, Y. Wang, O. Watson, P. Winskill, X. Xi, C. Whittaker, P. G. T. Walker, A. Ghani, C. A. Donnelly, S. Riley, L. C. Okell, M. A. C. Vollmer, N. M. Ferguson, S. Bhatt, Report 13: Estimating the number of infections and the impact of non-pharmaceutical interventions on COVID-19 in 11 European countries (Imperial College London, 2020); https://doi.org/10.25561/77731.

2. Public Health Institute of Sweden, Estimates of the peak-day and the number of infected individuals during the covid-19 outbreak in the Stockholm region, Sweden February-April 2020 [in Swedish] (2020); www. folkhalsomyndigheten.se/contentassets/2da059f90b90458d8454a0495 5d1697f/skattning-peakdag-antal-infekterade-covid-19-utbrottet-stockholmslan-februari-april-2020.pdf.

3. Instituto de Salud Carlos II, Second round national sero-epidemiology study of SARS-COV-2 infection in Spain [in Spanish] (2020);

https:/ www.mscbs.gob.es/ciudadanos/ene-covid/docs/ESTUDIO_ENECOVID19 SEGUNDA RONDA INFORME PRELIMINAR.pdf.

4. N. M. Ferguson, D. Laydon, G. Nedjati-Gilani, N. Imai, K. Ainslie, M. Baguelin, S. Bhatia, A. Boonyasiri, Z. Cucunubá, G. Cuomo-Dannenburg, A. Dighe, I. Dorigatti, H. Fu, K. Gaythorpe, W. Green, A. Hamlet, W. Hinsley, L. C. Okell, S. van Elsland, H. Thompson, R. Verity, E. Volz, H. Wang, Y. Wang, P. G. T. Walker, C. Walters, P. Winskill, C. Whittaker, C. A. Donnelly, S. Riley, A. C. Ghani, Report 9: Impact of nonpharmaceutical interventions (NPIs) to reduce COVID-19 mortality and healthcare demand (Imperial College London, 2020); https://doi.org/10.25561/77482.

5. W. Bock, B. Adamik, M. Bawiec, V. Bezborodov, M. Bodych, J. P. Burgard, T. Goetz, T. Krueger, A. Migalska, B. Pabjan, T. Ozanski, E. Rafajlowicz, W. Rafajlowicz, E. Skubalska-Rafajlowicz, S. Ryfczynska, E. Szczurek, P. Szymanski, Mitigation and herd immunity strategy for COVID-19 is likely to fail. medRxiv 2020.03.25.20043109 [Preprint]. 5 May 2020; https://doi.org/10.1101/2020.03.25.20043109.

6. H. Salje, C. T. Kiem, N. Lefrancq, N. Courtejoie, P. Bosetti, J. Paireau, A. Andronico, N. Hozé, J. Richet, C.-L. Dubost, Y. Le Strat, J. Lessler, D. Levy-Bruhl, A. Fontanet, L. Opatowski, P.-Y. Boelle, S. Cauchemez, Estimating the burden of SARS-CoV-2 in France. Science 10.1126/science.abc3517 (2020). doi:10.1126/science.abc3517

7. O. Diekmann, H. Heesterbeek, T. Britton, Mathematical Tools for Understanding Infectious Disease Dynamics (Princeton Univ. Press, 2013).

8. J. Wallinga, P. Teunis, M. Kretzschmar, Using data on social contacts to estimate age-specific transmission parameters for respiratory-spread infectious agents. Am. J. Epidemiol. 164, 936-944 (2006). doi:10.1093/aje/kwi317 Medline

9. R. Pastor-Satorras, A. Vespignani, Epidemic spreading in scale-free networks. Phys. Rev. Lett. 86, 3200-3203 (2001). do::10.1103/PhysRevLett.86.3200 Medline

10. R. M. Anderson, R. M. May, Infectious Diseases of Humans: Dynamics and Control (Oxford Univ. Press, 1991).
11. M. J. Ferrari, S. Bansal, L. A. Meyers, O. N. Bjørnstad, Network frailty and the geometry of herd immunity. Proc. Biol. Sci. 273, 2743-2748 (2006). doi:10.1098/rspb.2006.3636 Medline

12. F. Ball, T. Britton, O. Lyne, Stochastic multitype epidemics in a community of households: Estimation and form of optimal vaccination schemes. Math. Biosci. 191, 19-40 (2004). doi:10.1016/i.mbs.2004.05.001 Medline

13. M. G. M. Gomes, R. M. Corder, J. G. King, K. E. Langwig, C. Souto-Maior, J. Carneiro, G. Goncalves, C. Penha-Goncalves, M. U. Ferreira, R. Aguas, Individual variation in susceptibility or exposure to SARS-CoV-2 lowers the herd immunity threshold. medRxiv 10.1101/2020.04.27.20081893 [Preprint]. 21 May 2020; https://doi.org/10.1101/2020.04.27.20081893.

14. F. Ball, T. Britton, P. Trapman, Code for: Population heterogeneity and consequences for herd immunity to SARS-CoV-2, Version 1, Zenodo (2020); https://doi.org/10.5281/zenodo.3899252.

15. H. Andersson, T. Britton, Stochastic Epidemic Models and Their Statistical Analysis (Springer, 2000)

16. S. N. Ethier, T. G. Kurtz, Markov Processes: Characterization and Convergence (Wiley, 2009)

\section{ACKNOWLEDGMENTS}

Funding: TB and PT acknowledge financial support from the Swedish Research Council, grant 2015-05015 (TB) and 2016-04566 (PT). Author contributions: TB initiated and led the project. FB performed numerical computations and simulations. All three authors developed methodology and wrote and revised the manuscript. Competing interests: The authors declare no competing interests. Data and materials availability: No data are used and all code for numerics and figure production, including a description for the different programs, is accessible through Zenodo (14). This work is licensed under a Creative Commons Attribution 4.0 International (CC BY 4.0) license, which permits unrestricted use, distribution, and reproduction in any medium, provided the original work is properly cited. To view a copy of this license, visit https://creativecommons.org/licenses/by/4.0/. This license does not apply to figures/photos/artwork or other content included in the article that is credited to a third party; obtain authorization from the rights holder before using such material.

\section{SUPPLEMENTARY MATERIALS}

science.sciencemag.org/cgi/content/full/science.MSID/DC1

Materials and Methods

Figs. S1 to S3

Table S1

References $(15,16)$

MDAR Reproducibility Checklist

7 May 2020; accepted 18 June 2020

Published online 23 June 2020

$10.1126 /$ science.abc6810 


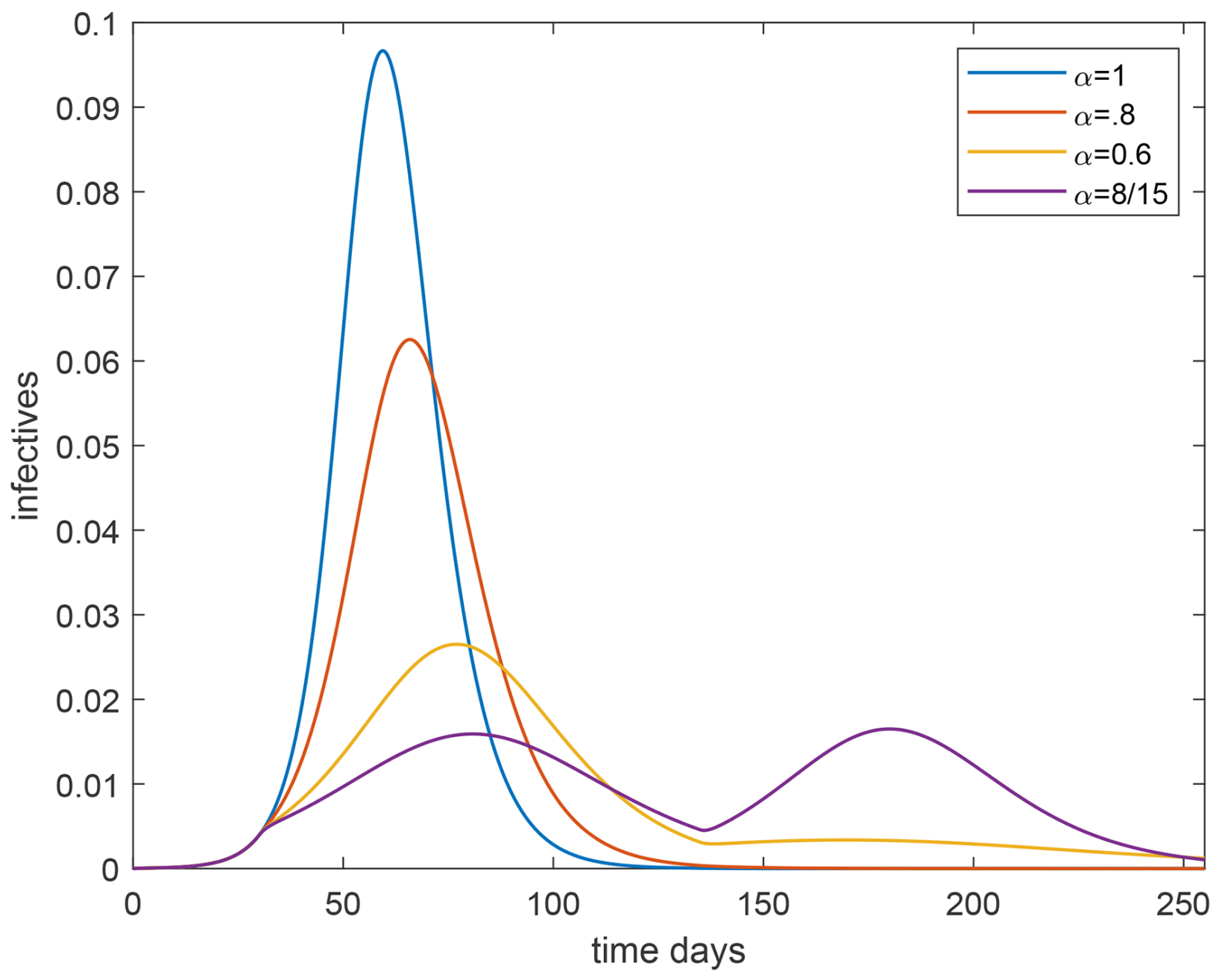

Fig. 1. Plot of the overall fraction infected over time for the age and activity structured community with $R_{0}=2.5$, for four different preventive levels inserted March 15 (day 30) and lifted June 30 (day 135). The blue, red, yellow and purple curves correspond to no, light, moderate, and severe preventive measures, respectively. 


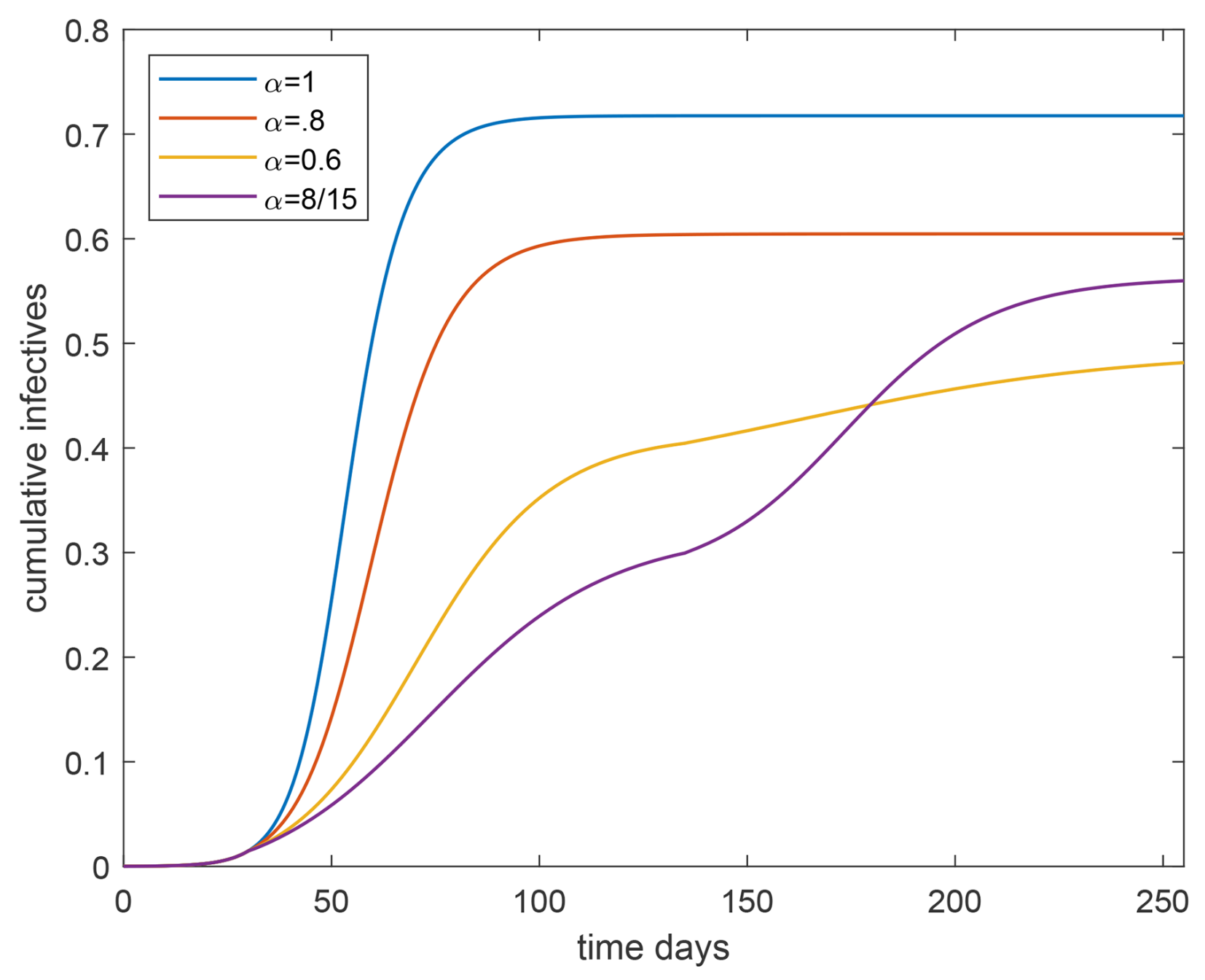

Fig. 2. Plot of the cumulative fraction infected over time for the age and activity structured community and $R_{0}=2.5$, for a four different preventive levels inserted March 15 and lifted June 30. The blue curve corresponds to no preventive measures, the red with light preventive measure, the yellow to moderate preventive measures and the purple corresponding to severe preventive measures. 
Table 1. Disease-induced herd immunity level $h_{\mathrm{D}}$ and classical herd immunity level $h_{\mathrm{C}}$ for different population structures. Numbers correspond to percentages.

\begin{tabular}{lcccccc}
\hline & \multicolumn{2}{c}{$R_{0}=2.0$} & \multicolumn{2}{c}{$R_{0}=2.5$} & \multicolumn{2}{c}{$R_{0}=3.0$} \\
Population structure & $h_{\mathrm{D}}$ & $h_{\mathrm{C}}$ & $h_{\mathrm{D}}$ & $h_{\mathrm{C}}$ & $h_{\mathrm{D}}$ & $h_{\mathrm{C}}$ \\
\hline Homogeneous & 50.0 & 50.0 & 60.0 & 60.0 & 66.7 & 66.7 \\
Age structure & 46.0 & 50.0 & 55.8 & 60.0 & 62.5 & 66.7 \\
Activity structure & 37.7 & 50.0 & 46.3 & 60.0 & 52.5 & 66.7 \\
Age and activity structure & 34.6 & 50.0 & 43.0 & 60.0 & 49.1 & 66.7 \\
\hline
\end{tabular}

Table 2. Final outcome fractions infected in different groups. Assuming $R_{0}=2.5$ and preventive measures put in place such that $\alpha=\alpha_{*}$ just barely reaching herd immunity for $R_{0}=2.5$. Population structure includes both age and activity and fractions infected are given as percentages.

\begin{tabular}{lccc}
\hline Age group & Low activity & Average activity & High activity \\
\hline 0-5 years & 17.6 & 32.1 & 53.9 \\
6-12 years & 25.8 & 44.9 & 69.7 \\
13-19 years & 31.4 & 52.9 & 77.8 \\
20-39 years & 27.4 & 47.2 & 72.1 \\
40-59 years & 22.8 & 40.3 & 64.4 \\
$\geq 60$ years & 14.6 & 27.0 & 46.7 \\
\hline
\end{tabular}

